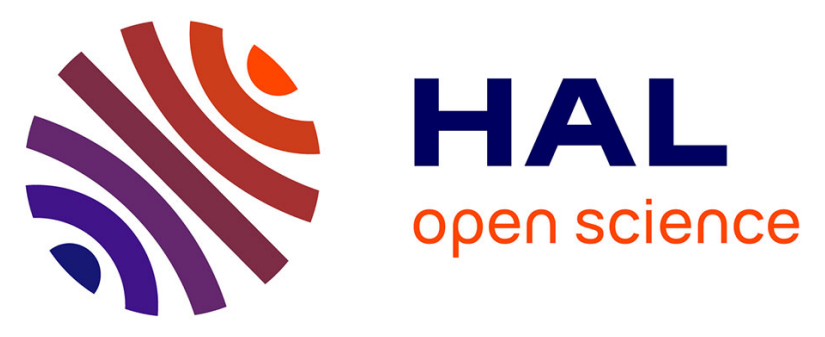

\title{
Does molecular docking reveal alternative chemopreventive mechanism of activation of oxidoreductase by sulforaphane isothiocyanates?
}

Pawel Mazur, Tomasz Magdziarz, Andrzej Bak, Zdzislaw Chilmonczyk, Teresa Kasprzycka-Guttman, Irena Misiewicz-Krzemińska, Katarzyna Skupińska, Jaroslaw Polanski

\section{To cite this version:}

Pawel Mazur, Tomasz Magdziarz, Andrzej Bak, Zdzislaw Chilmonczyk, Teresa Kasprzycka-Guttman, et al.. Does molecular docking reveal alternative chemopreventive mechanism of activation of oxidoreductase by sulforaphane isothiocyanates?. Journal of Molecular Modeling, 2009, 16 (7), pp.1205-1212. 10.1007/s00894-009-0628-5. hal-00568338

\section{HAL Id: hal-00568338 https://hal.science/hal-00568338}

Submitted on 23 Feb 2011

HAL is a multi-disciplinary open access archive for the deposit and dissemination of scientific research documents, whether they are published or not. The documents may come from teaching and research institutions in France or abroad, or from public or private research centers.
L'archive ouverte pluridisciplinaire HAL, est destinée au dépôt et à la diffusion de documents scientifiques de niveau recherche, publiés ou non, émanant des établissements d'enseignement et de recherche français ou étrangers, des laboratoires publics ou privés. 


\section{Editorial Manager(tm) for Journal of Molecular Modeling Manuscript Draft}

Manuscript Number: JMM0937R1

Title: Does molecular docking reveal alternative chemopreventive mechanism of activation of oxidoreductase by sulforaphane isothiocyanates?

Article Type: Original paper

Keywords: oxidoreductase, sulforaphane, indoquinoline, docking, molecular dynamics, isothiocyanates

Corresponding Author: Dr. Jaroslaw Polanski, Ph.D.,D.Sc.

Corresponding Author's Institution: University of Silesia

First Author: Jaroslaw Polanski, Ph.D.,D.Sc.

Order of Authors: Jaroslaw Polanski, Ph.D.,D.Sc.

Abstract: Isothiocyanates (ITC) are well-known chemopreventive agents extracted from vegetables. This activity results from the activation of human oxidoreductase. In this letter, the uncompetitive activatory mechanism of ITC was investigated using docking and molecular dynamics simulations. This indicates that $\mathrm{NAD}(\mathrm{P}) \mathrm{H}$ :quinone oxidoreductase can efficiently improve enzyme-substrate recognition within the catalytic site if the ITC activator supports the interaction in the uncompetitive binding site.

Response to Reviewers: Answers for reviews:

Reviewer \#2: The authors have explored uncompetitive activatory mechanism of isothiocyanates using docking and molecular dynamics simulations. Though the methodology adopted appears to be sound and discussion is convincing, how can they validate their hypothesis (either theoretical or experimental)?

Considering the suggestions we performed additional analyses to validate the hypothesis. It seems that the movements of several amino acids in the USB are constrained by the presence of the activator resulting in some changes of the CBS. Further testing of this hypothesis has been performed using the reduced dynamic approach (RedMD), which is based on the coarse-grained model of the protein. Thus, two representations of $\mathrm{NAD}(\mathrm{P}) \mathrm{H}$ :quinine oxidoreductase have been prepared by changing each amino acid with artificial alpha carbon atoms having appropriate properties to fake replaced amino acids. The first representation mimics the original 1D4A crystal structure, whereas the second one modifies the properties of ASN $45 \div 47$ of chain B and D, respectively. Generally, it reflects the constrains caused by the presence of activators in the UBS. Moreover, the applied modifications resulted in the increase of masses and harmonic constant. Afterward, both representations have been used in Langevin's dynamics simulations repeated 10 times with different random seeds. The whole trajectory time was $20 \mathrm{~ns}$ with temperature of $300 \mathrm{~K}$. The atomic coordinates of the system were sampled every $20 \mathrm{fs}$. Then, the entire set of the obtained frames has been superimposed by ASN $45 \div 47$ of chain B and D, respectively.

Fig. 5 in the revised version represents the resulting histograms of RMSD of alpha carbon (CA) of TYR128 calculated against its reference position taken from the original 1D4A file for the whole trajectory. The red color indicates TYR128 CA of the 1D4A reference structure, while the blue one 
shows TYR128 CA of the modified representations. Taking into account the imposed constraints on residues $45 \div 47$ minor but noticeable change of TYR128 CA behavior during the simulations might be noticed. The detailed analysis of the histogram revealed that TYR128 CA occupied approximately 5\% more time in the mean aberration. Additionally, it seems that TYR128 is less prone to the high deviation as it is shown in the subplot with logarithmic scale. The above conclusion might prove our previous observations. We observed that the constraints of $45 \div 47$ residues either by the presence of the activators or by manual modifications makes the position of TYR128 resulting from MD simulations more similar to its reference coordinates in the initial 1D4A file. Moreover, quite similar findings were obtained for HIST161, which plays pivotal role in the substrate binding in the CBS.

Reviewer \#3: In the paper of Polanski et al. the authors describe interesting MD and docking studies for alternative chemopreventive mechanism of activation of oxidoreductase by sulforaphane isothiocyanates.

Altogether they provide interesting results that might be of general relevance for anti-cancer research related to oxidoreductases.

Concerning the applied methods, the mixed force field approach (AMBER and MMFF94x) seems to be questionable to reflect true alterations in protein conformation. However, being aware of the difficulties for correct and rather expensive parameterization and partial charge assignment of ligands for consistent AMBER calculations the approach might be accepted as crude and fast approach.

3.a Nevertheless, the paper needs some essential improvements. In general, the paper is somewhat difficult to understand and follow for readers not directly involved in this field. E.g., a "simple" scheme showing the catalytic mechanisms exhibited by the enzyme would help.

Taking into account the comments we have improved the layout of "Result and discussion" section of our paper. We hope that this form of the paper will be better understandable for readers. A schematic plot introducing the catalytic mechanism exhibited by the enzyme has also been included as recommended.

3.b But much more relevant would be to show and explain, illustrated with corresponding detailed structural models (not only the interactions plots (Fig. 2 and 3) of the active site (or ligand binding site), what are the molecular reasons for the different Tyr128 conformational changes initiated by the ligands. Only then the paper will be of any value to gain new insights in structure function (activity) relationships.

Following your remarks some additional analyses have been carried out. It seems that the movements of several amino acids in the USB are constrained by the presence of the activator resulting in some changes of the CBS. Further testing of this hypothesis has been performed using the reduced dynamic approach (RedMD), which is based on the coarse-grained model of the protein. Thus, two representations of $\mathrm{NAD}(\mathrm{P}) \mathrm{H}$ :quinine oxidoreductase have been prepared by changing each amino acid with artificial alpha carbon atoms heaving appropriate properties to fake replaced amino acids. The first representation mimics the original 1D4A crystal structure, whereas the second one has modified properties of ASN $45 \div 47$ of chain B and D, respectively. Generally, it reflects the constrains caused by the presents of activators in the UBS. Moreover, the applied modifications resulted in the increase of masses and harmonic constant. Afterwards, both representations have been used in Langevin's dynamics simulations repeated 10 times with different random seeds. The whole trajectory time was $20 \mathrm{~ns}$ with temperature of $300 \mathrm{~K}$. The atomic coordinates of the system were sampled every $20 \mathrm{fs}$. Then, the entire set of the obtained frames has been superimposed by ASN $45 \div 47$ of chain B and D, respectively.

Fig. 5 in the revised version represents the resulting histograms of RMSD of alpha carbon (CA) of TYR128 calculated against its reference position taken from the original 1D4A file for the whole 
trajectory. The red color indicates TYR128 CA of the 1D4A reference structure, while the blue one shows TYR128 CA of the modified representations. Taking into account the imposed constraints on residues $45 \div 47$ minor but noticeable change of TYR128 CA behavior during the simulations might be noticed. The detailed analysis of the histogram revealed that TYR128 CA occupied approximately 5\% more time in the mean aberration. Additionally, it seems that TYR128 is less prone to the high deviation as it is shown in the subplot with logarithmic scale. The above conclusion might prove our previous observations. We observed that the constraints of $45 \div 47$ residues either by the presence of the activators or by manual modifications makes the position of TYR128 resulting from MD simulations more similar to its reference coordinates in the initial 1D4A file. Moreover, quite similar findings were obtained for HIST161, which plays pivotal role in the substrate binding in the CBS. 
Click here to download Abstract graphic: graphical.doc

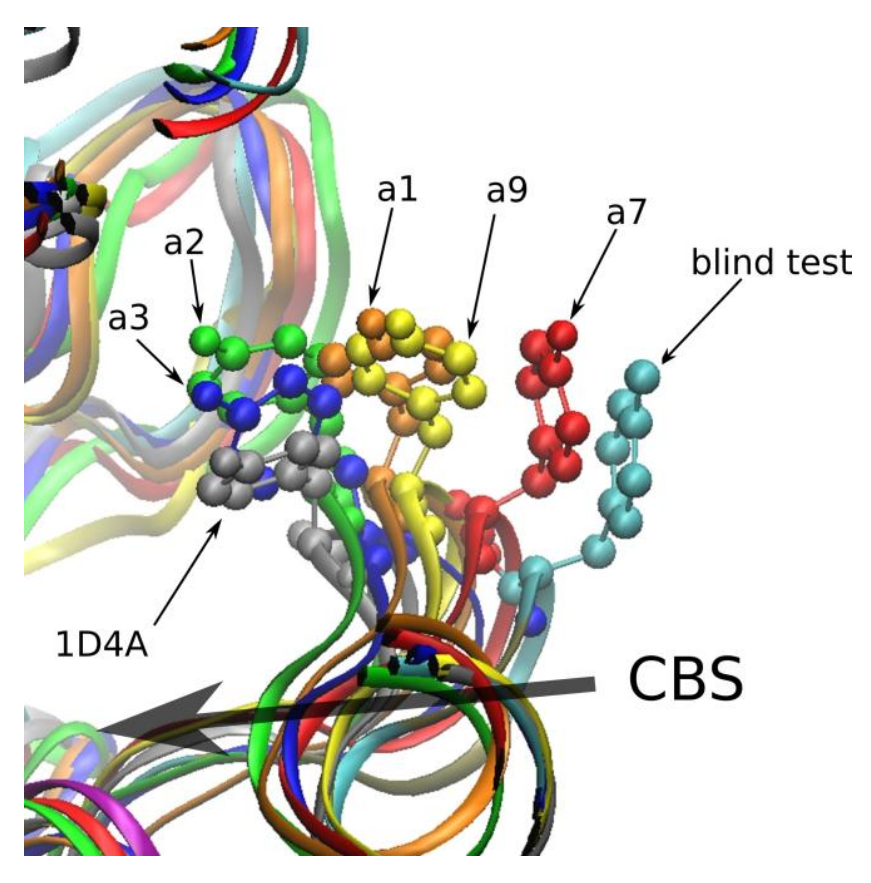




\title{
Does molecular docking reveal alternative chemopreventive mechanism of activation of oxidoreductase by sulforaphane isothiocyanates?
}

Received: 29.07.2009 / Accepted: 19.11.2009

Pawel Mazur ${ }^{1}$, Tomasz Magdziarz ${ }^{1}$, Andrzej Bak ${ }^{1}$, Zdzislaw Chilmonczyk ${ }^{1}$, Teresa Kasprzycka-Guttman ${ }^{2}$, Irena Misiewicz ${ }^{2}$, Jadwiga Skupińska ${ }^{2}$, and Jaroslaw Polanski ${ }^{1, \bigotimes}$

${ }^{1}$ Department of Organic Chemistry, Institute of Chemistry, University of Silesia, PL-40006 Katowice, Poland

${ }^{2}$ National Institute of Public Health, Chelmska 30/34, 00-725 Warszawa

${ }^{\varpi}$ Tel: +4832359 1128; Fax: +48 $32 \quad 259$ 9978; Email: polanski@us.edu.pl; http://www.chemoinformatyka.us.edu.pl/

\begin{abstract}
Isothiocyanates (ITC) are well-known chemopreventive agents extracted from vegetables. This activity results from the activation of human oxidoreductase. In this letter, the uncompetitive activatory mechanism of ITC was investigated using docking and molecular dynamics simulations. This indicates that $\mathrm{NAD}(\mathrm{P}) \mathrm{H}$ :quinone oxidoreductase can efficiently improve enzyme-substrate recognition within the catalytic site if the ITC activator supports the interaction in the uncompetitive binding site.
\end{abstract}

Keywords Oxidoreductase · Sulforaphane ' Indoquinoline · Docking · Molecular dynamics $\cdot$ Isothiocyanates 


\section{Introduction}

Over the last years there has been increasing interest in the role of sulforaphane (SP) compounds as cancer chemoprotective agents. SPs, phytochemical isothiocyanates (ITC), protected against chemically induced tumors in a variety of animal organs $[1,2]$. ITCs are found in a large number of edible plants, particularly those in the crucifer family [3-5]. The epidemiological studies have repeatedly shown that the consumption of these vegetables reduces the risk of many types of cancer in humans $[6,7]$. ITCs were found to elevate phase II enzyme activities, like glutathione S-transferases and DT diaphorase in a variety of cancer cell lines [8-10]. It was observed that ITCs blocking DNA damage by both inhibition of carcinogen activation through inhibition of phase I enzymes, mainly cytochromes P450 and detoxification of reactive carcinogens through induction of phase II enzymes. ITCs removed premalignant and malignant cells through the activation of apoptosis $[1,8,9]$, which has been shown to inhibit both initiation and post-initiation phases in animal models of chemical carcinogenesis [11-14] and the role of $\mathrm{NAD}(\mathrm{P}) \mathrm{H}$ :quinone oxidoreductase seems to be of key importance here.

Human $\mathrm{NAD}(\mathrm{P}) \mathrm{H}$ :quinone oxidoreductase is a homodimeric enzyme overexpressed in a variety of solid tumors, which makes it an interesting target for antitumor drugs. This enzyme plays a protective antioxidant role It catalyzes reduction of quinones to hydroquinones - see Fig. 1. At the same time it is capable of bioactivation of various prodrugs of the E09 or RH1 to their cytotoxic species. Several novel inhibitor series of this enzyme have been reported recently [15]. Moreover, a virtual screening was performed in the library of more than 700,000 molecules to identify potential $\mathrm{NAD}(\mathrm{P}) \mathrm{H}$ :quinone oxidoreductase ligands by molecular docking [16].

We have previously reported the synthesis of new ITC analogues activating $\mathrm{NAD}(\mathrm{P}) \mathrm{H}$ :quinone oxidoreductase $[17,18]$. We described also the differential response of human healthy lymphoblastoid and CCRF-SB leukemia cells to these compounds [18]. We also attempted to explain the induction of apoptosis by ITC in cell lines carrying various inherited BRCA1 mutations [19]. In the previous studies we were not able to indicate the molecular basis for ITC activation; however; the previously described mechanism of NQO1 activation involves signaling pathways Keap1/Nrf2/ARE and AhR/XRE. Thus, sulforaphane can bring about an increase in transcriptional activity resulting also in the increase of NQO1 level, which activates the enzyme activity [2]. 
On the other hand, we have recently observed that sulforaphane and its analogues can efficiently bind proteins, e.g. albumin, CYP1A1 and CYP1A2 [20]. We also observed that these molecules can be efficiently docked in the catalytic site of the oxidoreductase structure. However, an attempt to model quantitatively this mechanism by molecular docking within the binding site of the enzyme followed by the CoMSA analysis provided 3D QSAR model only slightly better than this resulted from the receptor independent ligand based superimposition [21].

Therefore, we attempted here to thoroughly probe the potential activation of the oxidoreductase by sulforaphane binding. The aim of present study was to use molecular docking to search for the alternative uncompetitive binding site (UBS) in the oxidoreductase. Thus, we used this method to observe the pattern of the distribution of the large population of ITC conformers within the enzyme. Basing on this pattern we decided to indicate the most highly populated site as a possible UBS. Then, using the molecular dynamic simulations (MDs) we attempted to reveal the influence of this effect on the hypothetical interaction of the second series compounds interacting with the oxidoreductase in the active site, as reported previously [15].

\section{Data sets and methodology}

The chemical structures of ITC analogues a1-a10 and their quinone oxidoreductase activation rate were reported according to the references $[17,18,22,23]$ and are shown in Table 1. Table 2 contains structures and inhibition potency data of a series of indoquinolinone reductase inhibitors (i1-i9) extracted from the literature $[15,24]$. This series is supplemented with diqumarol dq which is a known oxidoreductase inhibitor of the high activity [25]. Protomers, tautomers and conformers built for compounds a1-a10; i1-i9 and dq were optimized and partial atomic charges were calculated using the MMFF94x forcefield.

The structure of $\mathrm{NAD}(\mathrm{P}) \mathrm{H}$ :quinone oxidoreductase was taken from 1D4A PDB entry containing four amino acid chains complexed with four molecules of flavin adenine dinucleotide (FAD) [26]. The protein structure was prepared for docking as described previously. This included the addition of missing hydrogens, protein solvatation, the 
calculation of the atomic partial charges (AMBER99) and the protonation of the protein at physiological pH 7.4 using the PROPKA related method [27].

During docking a series of poses (ligand-protein complexes of particular conformation and mutual orientation) were generated for each molecule. The algorithm for the optimization of the ligand-protein orientation works by the alignment of triplets of ligand atoms on triplets of site points which are the centers of alpha spheres [28] created in the potential binding sites.

During the simulation the conformations were generated progressively from a single conformer (protomer, tautomer) by applying a collection of preferred torsions angles to the rotatable bonds. The quality of each alignment was further assessed by the London $\mathrm{dG}$ (LdG) scoring function (SF) which estimates the free energy of binding of the ligand and a set of the highest scored poses were chosen for each molecule (pose) docked [29].

Further investigation were completed using coarse-grained protein representation (reduced dynamics approach). We applied the one-bead force field for HIV-1 protease described previously by Tozzini et al. [30, 31] and Langevin dynamics [32, 33].

\section{Software}

Molecular modeling and molecular dynamics simulations were conducted using the Sybyl/Tripos and CCG MOE software packages and reduced molecular dynamics were conducted using ICM RedMD package running on the Intel Pentium PC with the GNU/Linux operating system.

\section{Results and discussion}

It was discovered and described in the literature that the catalytic core of quinone oxidoreductase is located in the vicinity of TYR128 [26]. We have reported previously that ITC molecules can be docked within this place, however, both the scoring functions for docking and the resulted 3D QSAR provided only poor quantitative models [21]. In order to reveal other possible binding sites for these compounds we generated alpha spheres all over the protein surface but in the catalytic binding site (CBS) (Fig. 2a). The docking provided 300 
poses scattered on the protein (Fig. 2b). The analysis of this indicates that the majority of poses $(>58 \%)$ concentrated in one site on the enzyme surface, in the proximity of the CBS. This suggests that the ITC activation mechanism may be of the uncompetitive nature involving the UBS (Fig. 2c). Additionally we tested some other potential UBSs $(>7 \%,>6 \%)$ using above procedure but the SF increase or interaction improvement was not noticed.

A visual inspection of activator-protein interactions in UBS allowed us to observe frequent interactions of activators a1-a10 with ASN45 and ASN47 residues. This involves the -NCS, CO-, -SO- ITC functionalities and can occur either directly or via water molecules (Fig. 3). As binding activator in the UBS could result in the CBS changes we attempted to model this effect using the MD simulations. Thus, prior to MDs, each activator-1D4A complex was minimized to energy gradient $0,01 \mathrm{kcal} \mathrm{mol}^{-1}$ and then molecular dynamics were performed using forcefields AMBER99 and MMFF94x with the Nosé-Poincaré-Andersen [34, 35] algorithm under constant temperature and volume. Although there were several controversies over the application of MMFF94 to MDs [36] we decided to use the MMFF94x variant in our simulations. MMFF94 is reported as the efficient forcefield for minimizing ligand-protein complexes [36, 37], furthermore, the MMFF94x variant ensures planar geometry of the peptide bonds [38]. Moreover, parametrization problems in AMBER99 made this approach unfeasible. The simulation time was $1 \mathrm{~ns}$ with the step $2 \mathrm{fs}$ under $300 \mathrm{~K}$. The partial charges (AMBER99) were recalculated after the MD simulation. In order to control possible artifacts we additionally performed a blind test using strictly the same protocol in which activatorenzyme structure was replaced by the enzyme without the activator. As these were expensive and time consuming processes we selected only several representative protein-activator complexes, namely, with compounds a1, a2, a3, a7, a9, a10. This selection was made on the basis of the activity values and structural diversity of the compounds. The results were analyzed by the RMSD values of the chain D and TYR128 in the 1D4A and the 1D4Aactivator complexes (Table 3), if compared to 1D4A in the initial and after MD blind test states. The main differences can be observed in the RMSD of TYR128. A large RMSD (TYR128) of $5.2 \AA$ exists between the 1D4A initial state and 1D4A after blind test. Similarly the large RMSD (TYR128) values describe the differences between the 1D4A-activator complexes in relation to the 1D4A after blind test. In contrary, the activator if present, makes the position of TYR128 in 1D4A after MD more similar to the position TYR128 in the initial 1D4A (lower values of TYR128 RMSD in 1D4A-activator in relation to 1D4A). The importance of the TYR128 in the substrate binding suggests that activator binding in UBS can affect substrate activity in CBS. Indeed, Fig. 4 shows changes in the TYR128 position as 
resulted in MDs discussed above. The most active activators (green and blue) bend the TYR128 loop into the CBS whereas the medium or low effective activators in UBS (blind test) causes the TYR128 loop bends outside the CBS. The latter effect can also be observed in the absence of the activator.

In order to test this hypothesis we performed further experiment in which the activator-ligand complexes and blind test systems as obtained form the MDs were used as the target for the hypothetical further docking of i1-i9 and dq in the CBS. The alignment process was done using alpha spheres located in the direct neighborhood of TYR128. Docking algorithm was essentially the same as in the case of activators though additional refinement step was performed to minimize each pose (MMFF94x) and then recalculate the SF. Table 4 presents the SF values for the best docked poses of each inhibitor with in the presence of activator in the UBS and for the blind test. The lower a value of the SF the better is a fit of the inhibitor. Thus, in the majority of cases the activator, if present, in the UBS, enhances binding as indicated by the decrease of the SF value for the substrates docked in the CBS. Only several activator/substrate/enzyme systems do not follow this rule. These are i2/a1; i10/a3, i9/a7 and i7/a10.

It seems that the movements of several amino acids in the USB are constrained by the presence of the activator resulting in some changes of the CBS. Further testing of this hypothesis have been performed using the reduced dynamic approach (RedMD), which utilizes the coarse-grained models of the proteins. Thus, two representations of $\mathrm{NAD}(\mathrm{P}) \mathrm{H}$ :quinine oxidoreductase have been prepared by changing each amino acid with artificial alpha carbon atoms heaving appropriate properties to fake replaced amino acids. The first representation mimics the original 1D4A crystal structure, whereas the second one has modified properties of ASN45 $\div 47$ of chain B and D, respectively. Generally, it reflects the constrains caused by the presents of activators in the UBS. Moreover, the applied modifications resulted in the increase of masses and harmonic constant. Afterward, both representations have been used in Langevin's dynamics simulations repeated 10 times with different random seeds. The whole trajectory time was 10 times $20 \mathrm{~ns}$ with temperature of $300 \mathrm{~K}$. The atomic coordinates of the system were sampled every $20 \mathrm{fs}$. Then, the entire set of the obtained frames has been superimposed by ASN $45 \div 47$ of chain B and D, respectively.

Fig. 5 represents the resulting histograms of RMSD of alpha carbon (CA) of TYR128 calculated against its reference position taken from the original 1D4A file for the whole 
trajectory. The red color indicates TYR128 CA of the 1D4A reference structure, while the blue one shows TYR128 CA of the modified representations. Taking into account the imposed constraints on residues $45 \div 47$ minor but noticeable change of TYR128 CA behavior during the simulations might be noticed. The detailed analysis of the histogram revealed that TYR128 CA occupied approximately 5\% more time in the mean aberration. Additionally, it seems that TYR128 is less prone to the high deviation as it is shown in the subplot with logarithmic scale. The above conclusion might prove our previous observations. We observed that the constraints of $45 \div 47$ residues either by the presence of the activators or by manual modifications makes the position of TYR128 resulting from MD simulations more similar to its reference coordinates in the initial 1D4A file. Moreover, quite similar findings were obtained for HIST161, which plays the pivotal role in the substrate binding in the CBS.

On the other hand we also observed the changes in behavior of other amino acids CAs. However, observed changes were of low importance.

In many recent papers authors claim that the scoring functions describing molecular docking experiments cannot be quantitatively correlated to biological activity [39]. Thus, in Fig. 6 we

presented the two-dimensional ligand-receptor interaction diagrams which allows one to qualitatively observe binding in the presence of activators. This depicts relatively strong connections or hydrogen bonds as well as electrostatic or charge-transfer interactions between a ligand and the respective amino acid residues. The comparison of the individual poses for the activator/enzyme (Fig. 6bc) with those resulted for the plane enzyme (Fig. 6a) indicates that the activator enables substrate to get easier in the catalytic core expanding a molecular volume inside receptor and simultaneously improves the solvent exposure of TYR128.

\section{Conclusions}

In conclusion the activatory effect on $\mathrm{NAD}(\mathrm{P}) \mathrm{H}$ :quinone oxidoreductase enzyme was investigated using molecular dynamics and molecular docking. This allowed us to find a hypothetical UBS for the series of ITC compounds. The ASN45 and ASN47 amino acid residues were recognized as the most probable binding residues in the UBS where ITC analogues were bound. Using the MDs for the enzyme/activator/substrate systems allowed us to indicate the enhancement of the substrate binding within the CBS, and thus directly 
simulate the protein activation effect. This effect can indicate an alternative mechanism of the oxidoreductase activation. We are now performing the series of experiments to prove this experimentally.

\section{Acknowledgments}

This study is supported by a grant from the Polish Ministry of Science N405 178735. PM thanks the UPGOW (in Polish Uniwersytet Partnerem Gospodarki Opartej na Wiedzy) grant founded by European Social Fund for partially financing his $\mathrm{PhD}$ studies. 


\section{References}

1. Lingxiang Y, Zang Y (2001) Total intracellular accumulation levels of dietary isothiocyanates determines their activity in elevation of cellular glutathione and induction of Phase 2 detoxification enzymes. Carcinogenesis 22:1987-1992

2. Kwak MK, Wakabayashi N, Kensler TW (2004) Chemoprevention through the Keap1Nrf2 signaling pathway by phase 2 enzyme inducers. Mutat Res 555:133-148

3. Chiao JW, Chung FL, Kancherla R, Ahmed T, Mittelman A, Conaway CC (2002) Sulforaphane and its metabolite mediate growth arrest and apoptosis in human prostate cancer cells. Int J Oncol 20:631-636

4. Iori R. Bernardi D, Gueyrard P, Rollin P, Palmieri S (1999) Formation of glucoraphanin by chemoselective oxidation of natural glucoerucin: a chemoenzymatic route to sulforaphane Bioorg Med Chem Lett 9:1047-1048

5. Shapiro T, Fahey JW, Wade L, Stephenson K, Talalay P (2001) Chemoprotective glucosinolates and isothiocyanates of broccoli sprouts: metabolism and excretion in humans.Cancer Epidemiol. Biomarkers Prev 10:501-508

6. Morimitsui Y, Nakagawa Y, Hayashi K, Fujii H, Kumaga TA (2002) A sulforaphane analogue that potently activates the Nrf2-dependent detoxification pathway. J Bio Chem $277: 3456-3463$

7. Herman A, Johnson DE, Singh V (2006) Sulforaphane causes autophagy to inhibit release of cytochrome $\mathrm{C}$ and apoptosis in human prostate cancer cells. Cancer Res 66:5828-5835

8. Lingxiang Y, Dinkova-Kostova T, Wade L, Zhang Y, Shapiro T, Talalay P (2002) Quantitative determination of dithiocarbamates in human plasma, serum, erythrocytes and urine: pharmacokinetics of broccoli sprout isothiocyanates in humans. Clin Chim Acta 316:43-53

9. Zhang, Y, Kolm RH, Mannervik B, Talalay P (1995) Reversible conjugation of isothiocyanates with glutathione catalyzed by human glutathione transferases. Biochem Biophys Res Commun 206:748-755

10. Zhang Y (2004) Cancer-preventive isothiocyanates: measurement of human exposure and mechanism of action. Mutat Res 555:173-190

11. Zhang Y (2001) Molecular mechanism of rapid cellular accumulation of anticarcinogenic isothiocyanates. Carcinogenesis 22:425-431 
12. Zhang Y, Talalay P (1998) Mechanism of differential potencies of isothiocyanates as inducers of anticarcinogenic Phase 2 enzymes. Cancer Res 58:4632-4639

13. Zhang Y, Talalay P, Cho CG, Posner GH (1992) A major inducer of anticarcinogenic protective enzymes from broccoli: isolation and elucidation of structure. Proc Natl Acad Sci USA 89:2399-2403

14. Jiang ZQ, Chen C, Yang B, Hebbar V, Kong AN.(2203) Differential responses from seven mammalian cell lines to the treatments of detoxifying enzyme inducers. Life Sci $72: 2243-2253$

15. Reigan P, Colucci MA, Siegel D, Chilloux A, Moody CJ, Ross D (2007) Development of indolequinone mechanism-based inhibitors of $\mathrm{NAD}(\mathrm{P}) \mathrm{H}$ :quinone oxidoreductase 1 (NQO1): NQO1 inhibition and growth inhibitory activity in human pancreatic MIA PaCa-2 cancer cells. Biochemistry 46:5941-5950

16. Nolan KA, Timson DJ, Stratford IJ, Bryce RA (2006) In silico identification and biochemical characterization of novel inhibitors of NQO1. Bioorg Med Chem Lett $16: 6246-6254$

17. Misiewicz I, Skupinska K, Kowalska E, Lubinski J Kasprzycka-Guttman T (2004) Sulforaphane-mediated induction of a phase 2 detoxifying enzyme NAD(P)H:quinone reductase and apoptosis in human lymphoblastoid cells. Acta Biochim Pol 51:711-721

18. Misiewicz I, Skupinska K Kasprzycka-Guttman T (2007) Differential response of human healthy lymphoblastoid and CCRF-SB leukemia cells to sulforaphane and its two analogues: 2-oxohexyl isothiocyanate and alyssin. Pharmacol Rep 59:80-87

19. Misiewicz I, Kozar A, Skupinska K, Kowalska E, Lubinski J, Kasprzycka-Guttman T (2005) Inhibition of cell cycle and induction of apoptosis by sulforaphane in cell lines carrying various inherited BRCA1 mutations. Oncol Rep 3:659-665

20. Kasprzycka-Guttman T, Misiewicz I, Skupińska K, Chilmonczyk Z, Polanski J, Mazur P, Magdziarz T, Bak A (2009) under preparation

21. Magdziarz T, Mazur P, Polanski J (2009) Receptor independent and receptor dependent CoMSA modeling with IVE-PLS: application to CBG benchmark steroids and reductase activators. J Mol Model 15:41-51

22. Posner GH, Cho CG, Green JV, Zhang Y, Talalay P (1994) Design and synthesis of bifunctional isothiocyanate analogs of sulforaphane: correlation between structure and potency as inducers of anticarcinogenic detoxication enzymes. J Med Chem 37:170-176 
23. Zhang Y, Kensler TW, Posner GH, Talalay P (1994) Anticarcinogenic activities of sulforaphane and structurally related synthetic norbornyl isothiocyanates. Proc Natl Acad Sci USA 91:3147-3150

24. Zhou Z, Fisher D, Spidel J, Greenfield J, Patson B, Fazal A, Wigal C, Moe OA, Madura JD (2003) Kinetic and docking studies of the interaction of quinones with the quinone reductase active site. Biochemistry 42:1985-1994

25. Cullen JJ, Hinkhouse MM, Grady M, Gaut AW, Liu J, Zhang Y, Darby Weyder CJ, Domann FE, Oberley LW (2003) Dicumarol inhibition of NADPH:quinone oxidoreductase induces growth inhibition of pancreatic cancer via a superoxide-mediated mechanism. Cancer Res 63:5513-5520

26. Faig M, Bianchet MA, Talalay P, Chen S, Winski S, Ross D, Amzel LM (2000) Structures of recombinant human and mouse NAD $(\mathrm{P}) \mathrm{H}$ :quinone oxidoreductases: species comparison and structural changes with substrate binding and release. Proc Natl Acad Sci USA 97:3177-3182

27. Li H, Robertson AD, Jensen JH (2005) Very fast empirical prediction and rationalization of protein pKa values. Proteins 61:704-721

28. Edelsbrunner H, Facello M, Fu R, Liang J (1995) Proceedings of the 28th Hawaii International Conference on Systems Science. pp 256

29. Molecular Operating Environment (MOE 2008.10) CCG Inc 1255 University St., Suite 1600, Montreal, Quebec, Canada H3B 3X3, http://www.chemcomp.com/

30. Tozzini V, McCammon JA (2005) A coarse grained model for the dynamics of flap opening in HIV-1 protease. Chem Phys Lett 413:123-128

31. Tozzini V, Trylska J, Chang CE, McCammon JA (2007) Flap opening dynamics in HIV1 protease explored with a coarse-grained model. J Struct Biol 157:606-615

32. Pastor, RW, Brooks BR, Szabo A (1988) An analysis of the accuracy of Langevin and molecular dynamics algorithms. Mol Phys 65:1409-1419

33. Pastor RW (1994) Techniques and applications of Langevin dynamics simulations. In: Luckhurst GR, Veracini CA (eds) The Molecular Dynamics of Liquid Crystals. Kluwer Academic, Dordrecht, The Netherlands

34. Sturgeon JB, Laird BB (2000) Symplectic algorithm for constant-pressure molecular dynamics using a Nosé-Poincaré thermostat. J Chem Phys 112:3474-3482

35. Bond SD, Leimkuhler BJ Laird BB (1999) The Nosé-Poincaré Method for Constant Temperature Molecular Dynamics. J Comput Phys 151:114-134 
36. Kaminski G, Jorgensen WL (1996) Performance of the AMBER94, MMFF94, and OPLS-AA Force Fields for Modeling Organic Liquids. J Phys Chem 100:18010-18013

37. Halgren T (1990) Maximally diagonal force constants in dependent angle-bending coordinates. II. Implications for the design of empirical force fields. J Am Chem Soc $112: 4710-4723$

38. Halgren T (1999) MMFF VI. MMFF94s option for energy minimization studies. J Comput Chem 20:720-729

39. Zentgraf M, Steuber H, Koch C, La Motta C, Sartini S, Sotriffer CA, Klebe G (2007) How Reliable Are Current Docking Approaches for Structure-Based Drug Design? Lessons from Aldose Reductase. Angew Chem Int Ed 46:3575-3578 
Table 1 ITC activators of $\mathrm{NAD}(\mathrm{P}) \mathrm{H}$ :quinone oxidoreductase. Data according to publications $[17,18,21,22]$

\begin{tabular}{lll}
\hline \multicolumn{2}{l}{ Compound Structure } & $\begin{array}{l}\text { Activity } \\
\text { pAC }\end{array}$ \\
\hline a1 & & -1.18 \\
a2 & $\mathrm{SCN}\left(\mathrm{CH}_{2}\right)_{5} \mathrm{CH}_{3}$ & 0.70 \\
a3 & $\mathrm{SCN}\left(\mathrm{CH}_{2}\right)_{4}(\mathrm{~S}=\mathrm{O}) \mathrm{CH}_{3}$ & 0.70 \\
a4 & $\mathrm{SCNCH}_{2}(\mathrm{C}=\mathrm{O}) \mathrm{CH}_{3}$ & -0.30 \\
a5 & $\mathrm{SCN}\left(\mathrm{CH}_{2}\right)_{4}(\mathrm{~S}=\mathrm{O})\left(\mathrm{CH}_{2}\right)_{3} \mathrm{CH}_{3}$ & 0.40 \\
a6 & $\mathrm{SCN}\left(\mathrm{CH}_{2}\right)_{4}(\mathrm{~S}=\mathrm{O}) \mathrm{CH}_{3}$ & -0.45 \\
a7 & $\mathrm{SCN}\left(\mathrm{CH}_{2}\right)_{4}(\mathrm{C}=\mathrm{O}) \mathrm{SCH}_{3}$ & -0.45 \\
a8 & $\mathrm{SCN}\left(\mathrm{CH}_{2}\right)_{4}(\mathrm{C}=\mathrm{O}) \mathrm{OCH}_{3}$ & -0.30 \\
a9 & $\mathrm{SCN}\left(\mathrm{CH}_{2}\right)_{4} \mathrm{CN}$ & -0.20 \\
a10 & $\mathrm{SCN}\left(\mathrm{CH}_{2}\right)_{5}(\mathrm{~S}=\mathrm{O}) \mathrm{CH}_{3}$ & 0.30
\end{tabular}


Table 2 Indoquinoline inhibitors of reductase. Data according to reference [15]

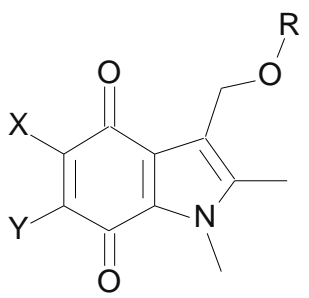

\begin{tabular}{|c|c|c|c|}
\hline \multirow[t]{2}{*}{ Com } & \multicolumn{2}{|c|}{ Structure } & $\begin{array}{l}\text { Activity } \\
\mathrm{IC}_{50}[\mu M]\end{array}$ \\
\hline & $\bar{X}$ & $\mathrm{R}$ & $4 \mathrm{~h} 72 \mathrm{~h}$ \\
\hline i1 & $\mathrm{OMe}$ & p-nitrophenyl & 10.630 .51 \\
\hline i2 & $\mathrm{H}$ & OMe p-nitrophenyl & 0.640 .35 \\
\hline i3 & $\mathrm{OMe}$ & phenyl & 1.390 .96 \\
\hline i4 & $\mathrm{H}$ & OMe phenyl & 4.560 .41 \\
\hline i5 & $\mathrm{OMe}$ & 4-pirydyl & 2.012 .17 \\
\hline i6 & $\mathrm{H}$ & OMe 4-pirydyl & 2.563 .12 \\
\hline i7 & $\mathrm{OMe}$ & H acetyl & 2.422 .16 \\
\hline i8 & $\mathrm{H}$ & OMe H & 2.151 .99 \\
\hline i9 & $\mathrm{OMe}$ & $\mathrm{H}$ & 2.172 .16 \\
\hline
\end{tabular}


Table 3 RMSD ( $⿱$ ) values of $\alpha$ carbons of chain D and TYR128. Two references were used, the 1D4A plain enzyme model and the blind test model resulted in MD. Details in text

\begin{tabular}{lllll}
\hline \multirow{2}{*}{$\begin{array}{l}\text { 1D4A } \\
\text { model }\end{array}$} & $\begin{array}{l}\text { Chain D } \\
\text { In relation } \\
\text { to 1D4A }\end{array}$ & $\begin{array}{l}\text { In relation } \\
\text { to blind } \\
\text { test }\end{array}$ & $\begin{array}{l}\text { In relation } \\
\text { to 1D4A }\end{array}$ & $\begin{array}{l}\text { In relation } \\
\text { to blind } \\
\text { test }\end{array}$ \\
\hline blind test & 1.3 & $(0)$ & 5.2 & $(0)$ \\
with a1 & 1.3 & 1.5 & 2.3 & 4.2 \\
with a2 & 1.3 & 1.5 & 1.3 & 5.6 \\
with a3 & 1.2 & 1.3 & 1.2 & 4.1 \\
with a7 & 1.4 & 1.2 & 4.0 & 2.0 \\
with a9 & 1.5 & 1.4 & 2.5 & 3.7 \\
with a10 & 1.4 & 1.2 & 2.2 & 4.6
\end{tabular}


Table 4 The LdG scoring functions of best docked poses of the indoquinolinone inhibitors depending of the presence of activator and the binding site. The blind test was performed using all steps applied in the activity-mechanism simulations

\begin{tabular}{|c|c|c|c|c|c|c|c|}
\hline \multirow{2}{*}{ Inhibitor } & \multicolumn{7}{|c|}{ London dG scoring functions between the enzyme and enzyme/activator/substrate ${ }^{a /}$} \\
\hline & $1 \mathrm{D} 4 \mathrm{~A}^{\mathrm{b} /}$ & with a $1^{\mathrm{c}}$ & With a $2^{\mathrm{cl}}$ & with a3 ${ }^{\mathrm{cl}}$ & with a7 ${ }^{\mathrm{cl}}$ & with $\mathrm{a} 9^{\mathrm{c}}$ & with a10 ${ }^{\mathrm{c}}$ \\
\hline$\overline{\mathrm{i} 1}$ & -13.75 & -19.03 & -17.95 & -20.70 & -15.33 & -19.69 & -16.62 \\
\hline $\mathrm{i} 2$ & -14.08 & -13.89 & -18.25 & -18.78 & -14.44 & -20.31 & -19.32 \\
\hline i3 & -12.39 & -18.69 & -17.19 & -14.81 & -14.88 & -18.52 & -16.23 \\
\hline i4 & -12.06 & -16.31 & -15.58 & -18.09 & -13.14 & -17.88 & -20.25 \\
\hline i5 & -12.45 & -17.28 & -15.84 & -19.03 & -13.93 & -18.83 & -17.69 \\
\hline i6 & -11.36 & -17.70 & -15.21 & -16.24 & -12.54 & -17.68 & -19.60 \\
\hline i7 & -11.64 & -17.07 & -15.88 & -16.30 & -11.73 & -17.06 & -8.86 \\
\hline i8 & -11.71 & -17.00 & -12.84 & -17.70 & -11.85 & -14.88 & -15.81 \\
\hline i9 & -13.32 & -15.64 & -13.81 & -17.18 & -11.44 & -15.75 & -16.56 \\
\hline $\mathrm{dq}$ & -11.41 & -14.78 & -18.78 & -12.01 & -15.72 & -19.97 & -16.87 \\
\hline
\end{tabular}




\section{Figure captions}

Fig. 1 Schematic reaction catalyzed by Human NAD(P)H:quinone oxidoreductase

Fig. 2 A search for the UBS by molecular docking. The first step was to generate alpha spheres all over the protein surface but in the CBS (a); the docking resulted in 300 poses scattered on the protein $(\mathbf{b})$; the majority of poses $(>57 \%)$ gathers within the yellow circle (c) which reveals the potential UBS. Details in text

Fig. 3 Interaction plot illustrating a binding of the activator a2 in the UBS. Two interactions with ASN45 and ASN47 residues via water molecules are displayed involving -NCS and -SO- functionalities. Brown lines denote solvent contact interactions, blue areas on ligand atoms and blue shadow of amino acid residues represent ligand and receptor exposures respectively

Fig. 4 The changes of the position of TYR128 residues at the opening to the CBS as resulted from the activator binding in the UBS, as simulated by MD. Colors codes are as follows: gray - 1D4A plane enzyme; cyan - blind test; red - with a7; yellow - with a9; orange - with a1; green - with a2; blue - with a3. Details in text

Fig. 5 Histograms of RMSD of alpha carbon (CA) of TYR128 of the referenced (red) and modified (blue) structures calculated against its reference position taken from the original 1D4A file for the all 10 trajectories - nrf denotes number of frames; subplot in logarithmic scale

Fig. 6 Interaction plots illustrating a binding of inhibitor i2 without any activator (the blind test) (a), in the presence of a3 (b) and in the presence of a10 (c) activators. If the activator is present (b and $\mathbf{c}$ ) the direct interactions with TYR128 as well as more frequent indirect interactions via water molecules with other residues can be observed. Brown lines denote solvent contact interactions, green arrow ended lines denotes sidechain hydrogen bond donors, blue areas on ligand atoms and blue shadow of amino acid residues represent ligand and receptor exposures respectively 
Click here to download high resolution image

$\left.\right|_{0} ^{1}$

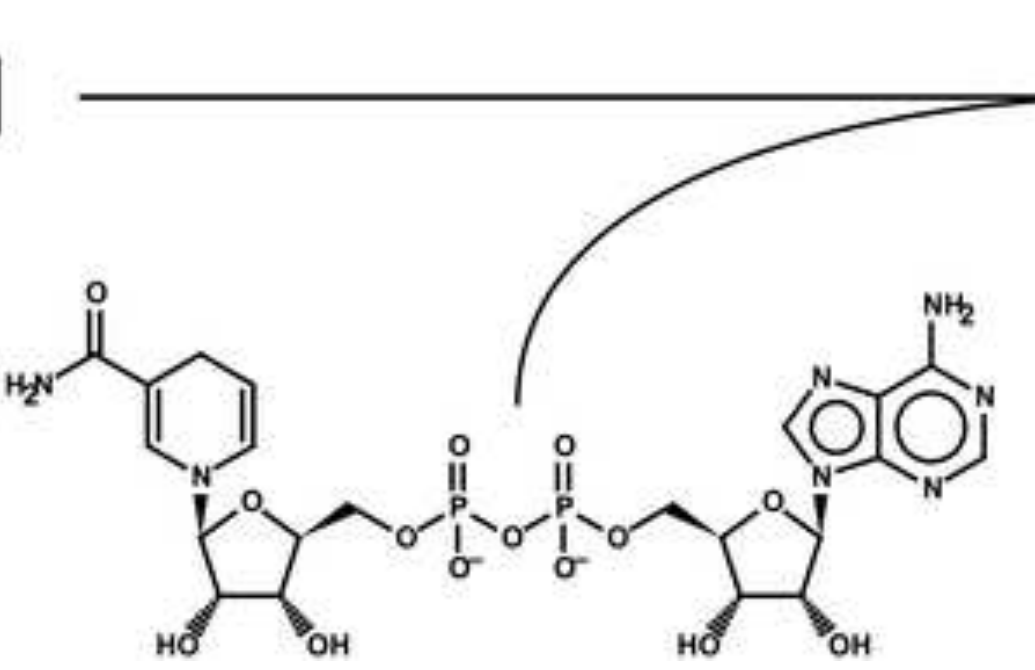

$\mathrm{H}^{+}$

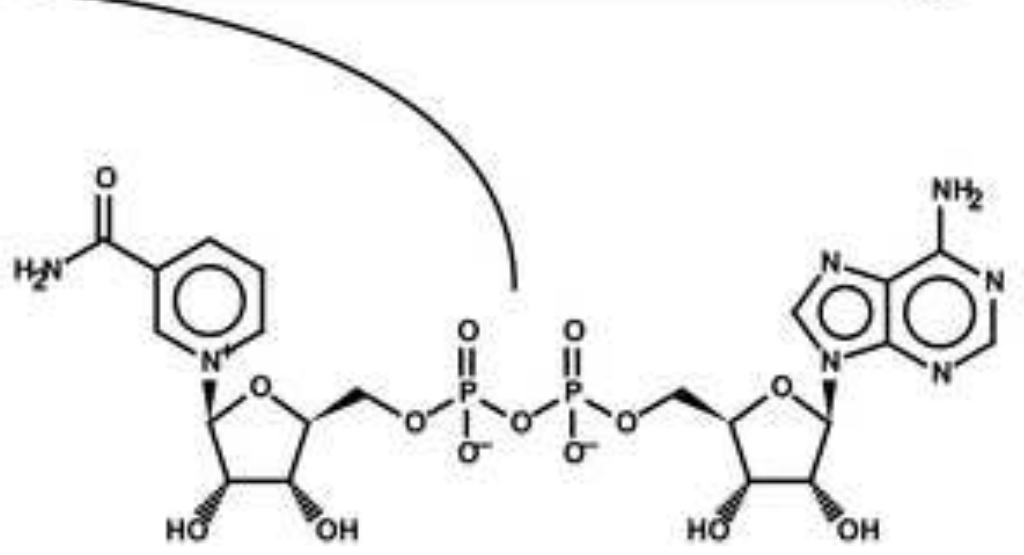

OH 
Figure 2
Click here to download high resolution image
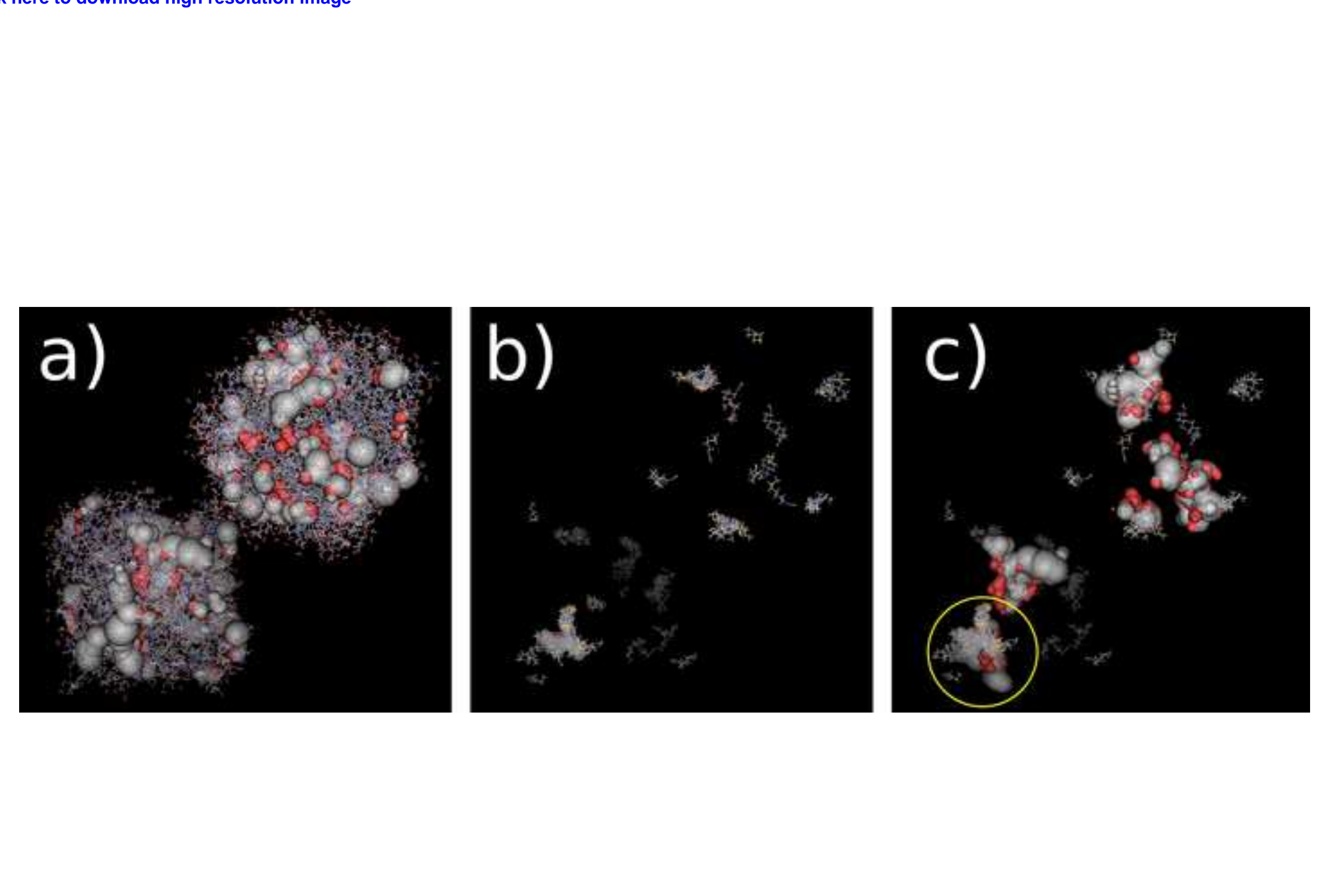

.

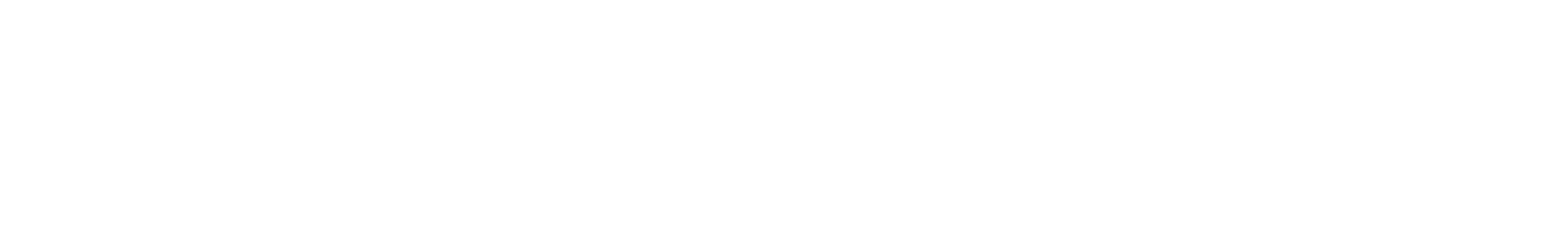

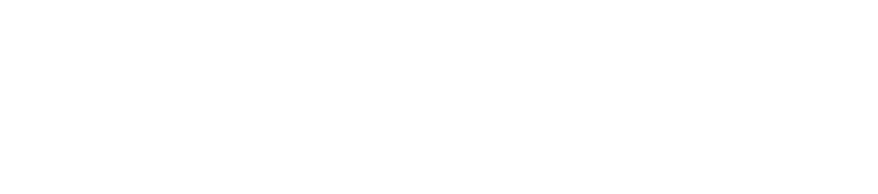


Click here to download high resolution image

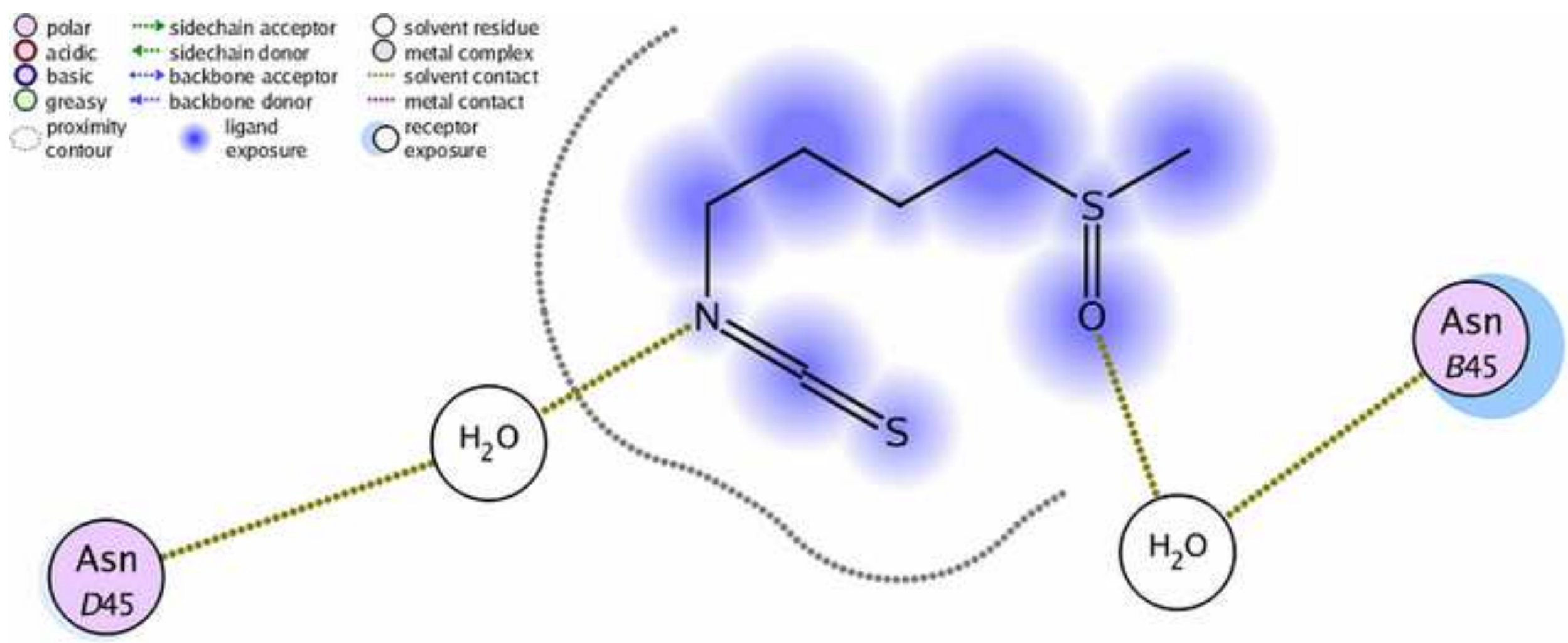


Click here to download high resolution image

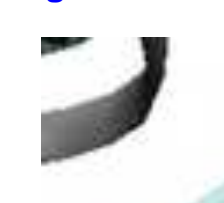

a1 a9
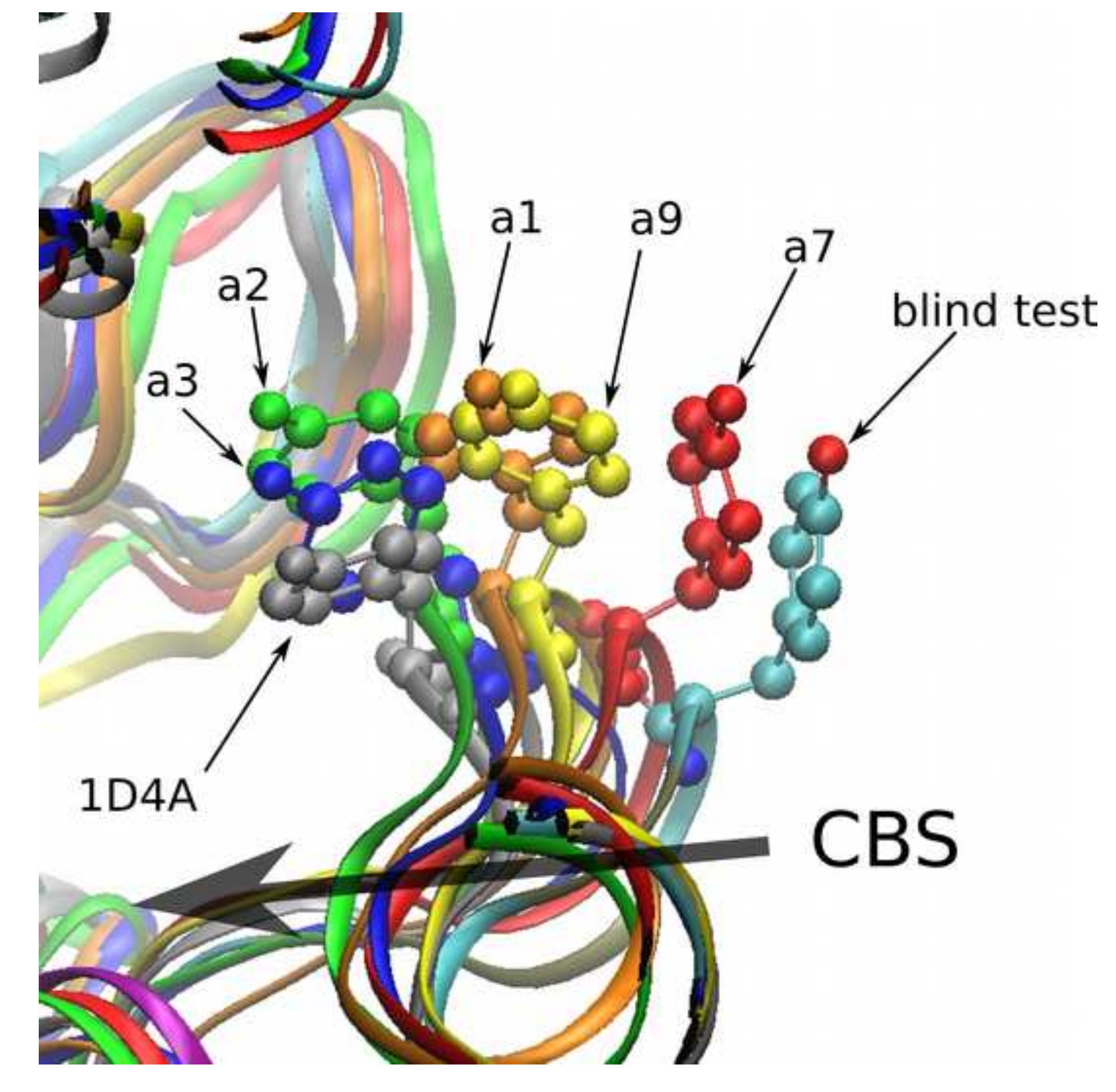


\section{Figure 5}

Click here to download high resolution image

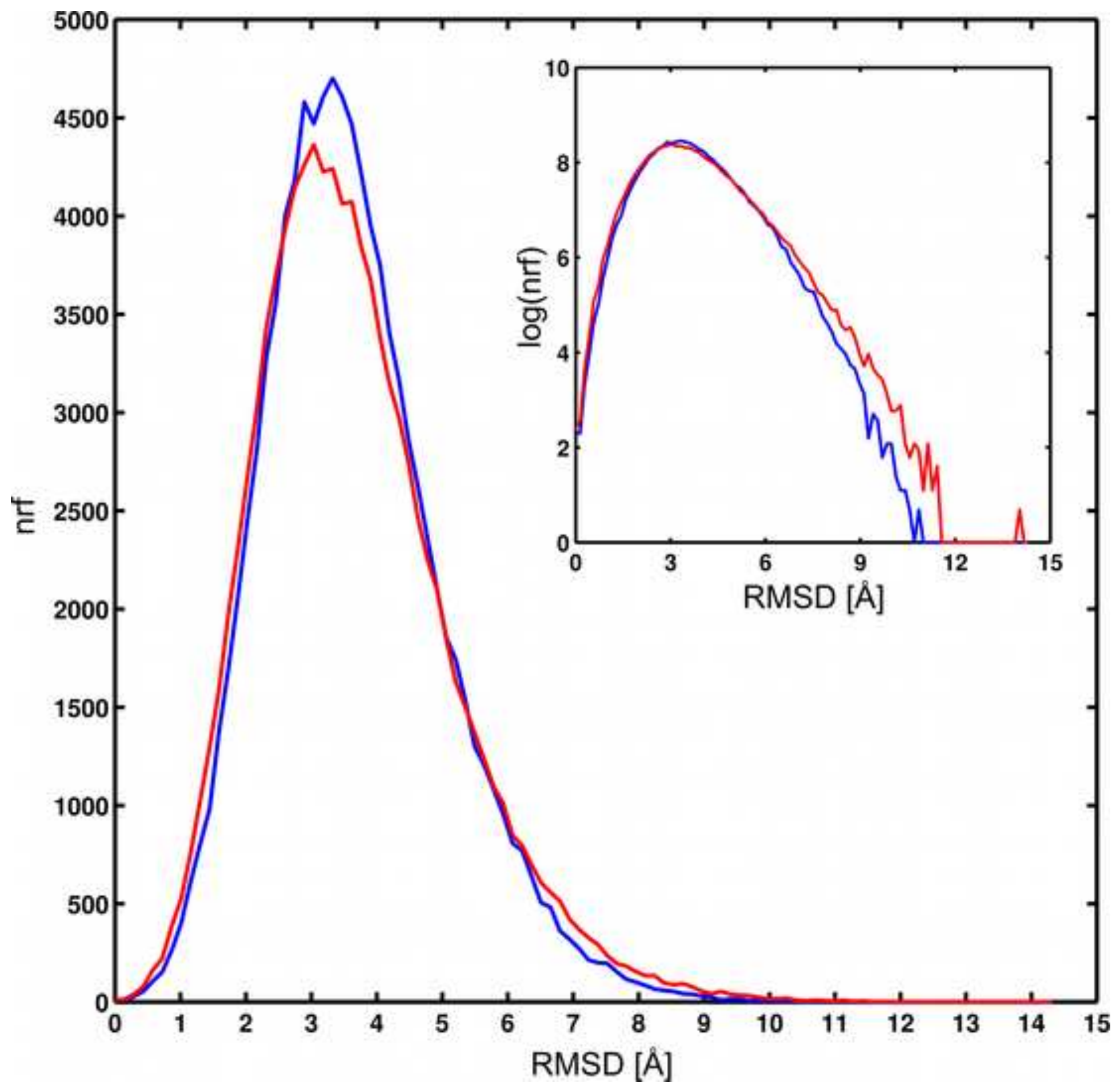



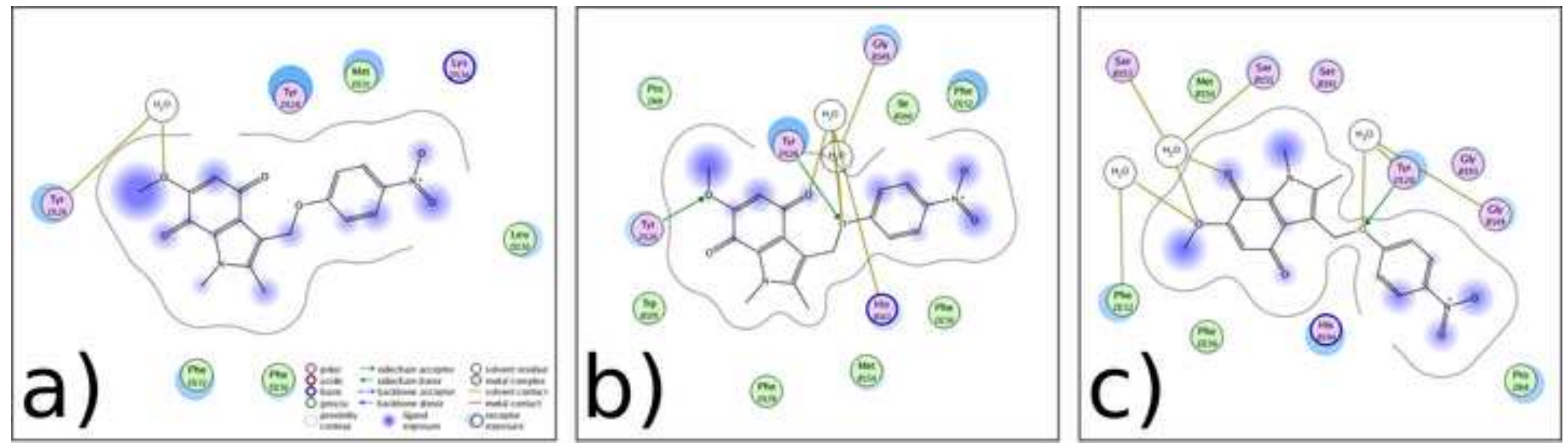

a)

(iii)

(요요 $8=8=$

b)

C) 